\title{
Charge Transport in C60 Crystals: A Tight-Binding Description
}

\author{
1,* Marcelo Lopes Pereira Junior (PG) and ${ }^{2}$ Luiz Antônio Ribeiro Júnior (PQ) \\ ${ }^{1}$ Institute of Physics, University of Brasília, Brasília, 70910-970, DF, Brazil, ${ }^{2}$ International Center for \\ Condensed Matter Physics, University of Brasília, P.O. Box 04531, 70.919-970, Brasília, DF, Brazil \\ Keywords: Charge Transport, Molecular Crystals, Polaron, C60.
}

\section{Introduction}

Organic Semiconductors have attracted much attention since the discovery of their charge transport properties [1]. Unique features such as light weight and flexibility make them interesting for the electronics industry, particularly when new green energy solutions are considered. Since charge transport is the key step behind the performance of optoelectronic devices based in molecular crystals, a deep understanding about the charge carrier properties at a molecular scale is fundamental. Here, a numerical study is carried out to investigate the polaron transport in C60 crystals using a semi-empirical Holstein-Peierls model [2]. Importantly, the results presented here may throw new light on the description of the polaron properties in C60-based crystals.

\section{Methodology}

The model Hamiltonian adopted here considers both intra and intermolecular electron-lattice interactions for describing a two-dimensional array (single layer) of molecules [2]. Each molecule in the array is defined as a site $\boldsymbol{i}, \boldsymbol{j}$ with three degrees of freedom: two antisymmetric non-local and phonon modes and one local phonon mode [2]. In our molecular array, the index $\boldsymbol{j}$ (x-direction) denotes the "columns" whereas $\boldsymbol{i}$ (y-direction) labels the "rows". The model Hamiltonian adopted in this work -- that incorporates the Holstein and Peierls contributions in a single Hamiltonian -- follows the expression presented in the reference [2]. The set of model parameters, used to describe the $\mathrm{C} 60$ crystal, is originally developed in the present work.

\section{Results}

In our calculations, we have obtained for the polaron formation energy -- that represents the difference between the energies for systems in the neutral ground state and in a relaxed configuration due to the presence of an extra electron or hole -- a value about $50 \mathrm{meV}$, which lies in the range of values reported for other classes of molecular crystals [2]. Such a result indicates that the our set of model parameters can be describe the polaron properties for $\mathrm{C} 60$ crystals in a realistic fashion.

Regarding the charge carrier dynamics in the C60 systems, in our model, from the influence of external electric field, in a simulation $5 \mathrm{ps}$, the polaron moves approximately $25 \AA$ from the origin, at a speed of approximately $5 \AA / \mathrm{ps}$. The electric field used here has an intensity of $2.0 \mathrm{mV} / \AA$. These results are presented in Figure 1. 


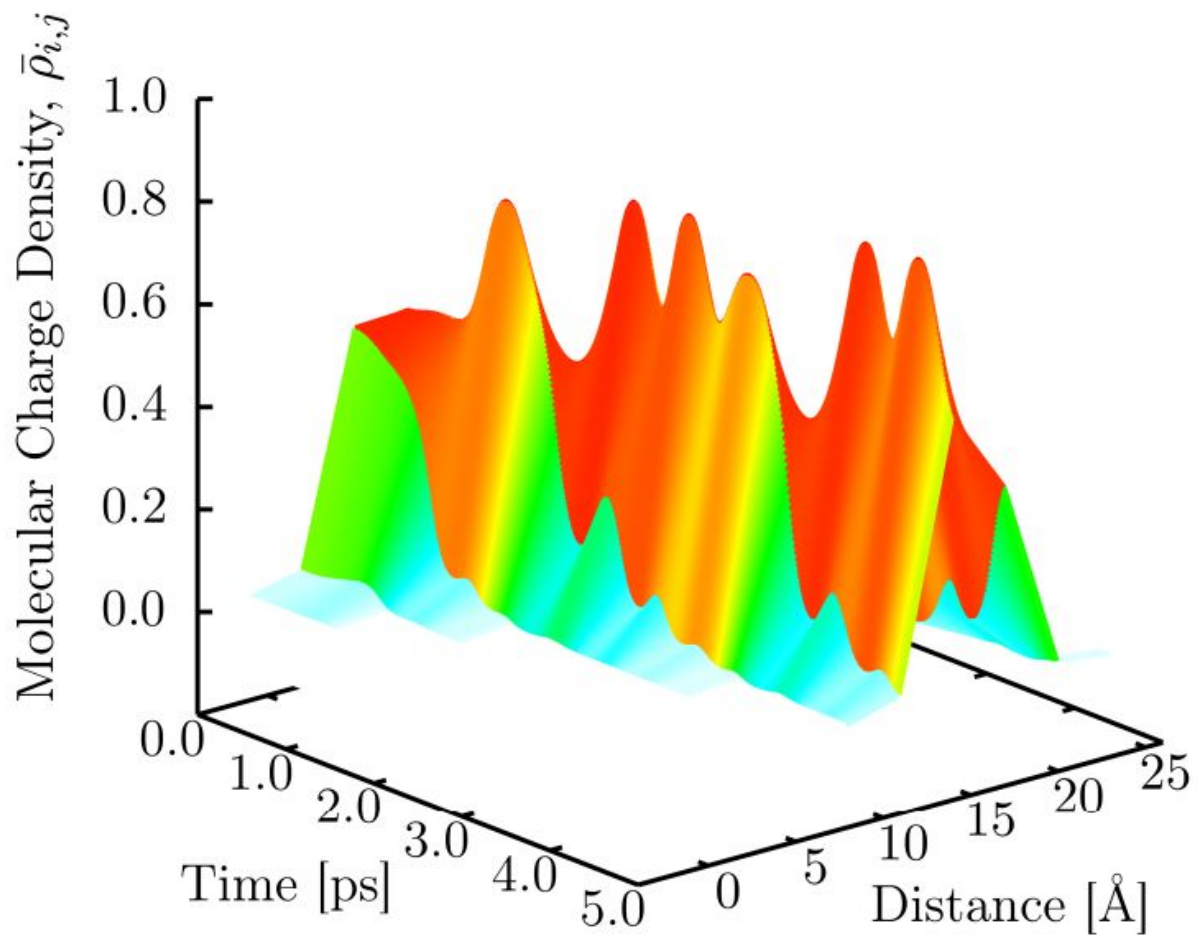

Figure 1. Dynamics of Molecular Charge Density on a C60 Crystal.

\section{Conclusions}

The results showed that a suitable set of parameters, that is employed in our model Holstein-Peierls Hamiltonian, can play the role of distinguishing the properties related to the polaron stability and dynamics in different organic molecular crystals.

\section{Acknowledgements}

The authors gratefully acknowledge the financial support from the Brazilian Research Councils CAPES and FAPDF. L.A.R.J. gratefully acknowledges the financial support from the Brazilian Research Council FAPDF grant 0193.000942/2015 and 193.001.511/2017. L.A.R.J. also wishes to thank the Brazilian Ministry of Planning, Budget and Management (Grant DIPLA 005/2016).

\section{References}

[1] V. Coropceanu, et. al. Chem. Rev., 107, 926-952, 2007.

[2] L. A. Ribeiro and Sven Stafström. Phys. Chem. Chem. Phys., 17, 8973-8982, 2015. 